\title{
CARACTERIZAÇÃO FÍSICO-MORFOLÓGICA DE FRUTOS DE MICROTANGERINAS (Citrus spp.) DE POTENCIAL UTILIZAÇÃO COMO PORTA-ENXERTOS ${ }^{1}$
}

\author{
JOSÉ RIBAMAR GUSMÃO ARAUJO² \& ARY APPARECIDO SALIBE ${ }^{3}$
}

\begin{abstract}
RESUMO - O presente trabalho objetivou caracterizar espécies e variedades de Citrus do grupo das microtangerinas de valor potencial como portaenxertos, de modo a propiciar maior conhecimento desse grupo de plantas e oferecer subsídios para futuras pesquisas. Um total de 14 variedades/ clones foram descritas, incluindo as seguintes espécies: C. reshni Hort. ex Tan., C. sunki Hort. ex Tan, C. pectinifera Tan., C. crenatifolia Lush., $C$. amblycarpa Och., C. tachibana Tan., C. lycopersicaeformis Hort. ex Tan., C. keraji Hort. ex Tan. e C. reticulata Blanco. A pesquisa foi conduzida no Departamento de Horticultura da FCA-Unesp, Botucatu-SP e os frutos foram obtidos dos BAGs de Citros da FCA-Unesp-SP, e do CCSM-IAC, Cordeirópolis-SP. Descritores físicos e morfológicos de frutos indicaram diferenças entre espécies/variedades. As microtangerinas apresentaram características semelhantes quanto ao pequeno tamanho dos frutos, forma oblata e coloração laranja dos frutos. C. amblycarpa, $C$. sunki e C. tachibana produziram os frutos de menor tamanho e espessura de casca. Elevado número de sementes por fruto foi encontado nas tangerinas 'Cleópatra', 'Sun Chu Sha Kat', 'Suen Kat' e 'Heennaran', enquanto 'Sunki' apresentou elevado número de sementes abortadas. Parece discutível a posição botânica de 'Suen Kat', ao que se propõe considerar a mesma como uma variedade de C. sunki.
\end{abstract}

Termos para indexação: Citrus spp., Microtangerinas, frutos, caracterização física, morfologia.

\section{PHYSICAL AND MORPHOLOGICAL CHARACTERIZATION OF FRUITS OF SMALL-FRUITED MANDARINS (Citrus spp.) OF POTENTIAL USE AS ROOTSTOCK}

\begin{abstract}
The present research aimed to characterize physical and morphological features of fourteen species/varieties of small-fruited mandarins of Tanaka's groups (Citrus spp.), and hence select the most promising types. It was described the followings species: $C$. reshni Hort. ex Tan., $C$. sunki Hort. ex Tan, C. pectinifera Tan., C. crenatifolia Lush., C. amblycarpa Och., C. tachibana Tan., C. lycopersicaeformis Hort. ex Tan., C. keraji Hort. ex Tan. and C. reticulata Blanco. The work was carried out at the Department of Horticulture/FCA-Unesp, Botucatu, SP, Brazil. Centers of Citrus Germoplasm Bank from FCA-Unesp and CCSM-IAC provided fruit materials. Physical and morphological fruits descriptors pointed out differences between varieties. This mandarins group presented as common characteristics their small size of fruits, oblate form and orange color. $C$. amblycarpa, C. sunki and C. tachibana produced the fruits of the smallest size. 'Cleopatra', 'Sun Chu Sha Kat', 'Suen Kat' and 'Heennaran' presented high number of seeds per fruit, while 'Sunki' presented high number of sterile seeds. It is discussed the botanical position of 'Suen Kat', which should be considered as a variety of $C$. sunki.
\end{abstract}

Index terms: Citrus spp., small-fruited mandarins, fruits, physical characterization, morphology.

\section{INTRODUÇÃO}

As plantas cítricas são originárias das regiões úmidas tropicais e subtropicais do continente asiático e ilhas adjacentes. No Brasil, as citrinas foram introduzidas pelos portugueses por volta de 1540, provavelmente na Bahia (Moreira \& Moreira, 1991).

A citricultura é atualmente uma importante atividade socioeconômica, em que a cultura comercial tem por base a muda enxertada, que está alicerçada sobre um número muito reduzido de portaenxertos, com ampla predominância do limoeiro- 'Cravo', cuja participação nos pomares pode variar desde 70 até $100 \%$. A preferência pela referida espécie está relacionada à sua ampla adaptação às variações de solo e clima e combinação com a maioria das variedades-copa, induzindo grande produção de frutos de boa qualidade. Seria um ideótipo como porta-enxerto, não fosse sua suscetibilidade ao 'declínio dos citros', de causa desconhecida, e à gomose de Phytophthora.

Levantamento realizado em viveiros do Estado de São Paulo pelo Fundecitrus - Fundo de Defesa da Citricultura, em 1998, revela que a situação praticamente não se alterou: o limoeiro- 'Cravo' lidera com $81,2 \%$ das plantas, seguido pela tangerineira- 'Cleópatra' (8,3\%), citrumelo 'Swingle' (5,9\%) e tangerineira-'Sunki' (2,2\%), que somados correspondem a 97,6\% do total de variedades (Salva,1998 - Informação Pessoal).

A grande maioria dos cultivos de citros no mundo esteve por muito tempo assentada sobre um número muito reduzido de porta-enxertos: a laranjeira- 'Azeda' Citrus aurantium L., o limoeiro-'Rugoso-daFlórida' C. jambhiri Lush., o limoeiro- 'Cravo' C. limonia Osbeck, e o 'Trifoliata' Poncirus trifoliata Raf. (Salibe, 1978; Wutscher, 1979).

A importância do porta-enxerto para a citricultura, segundo Salibe (1978), traduz-se pelas inúmeras influências e modificações que ele exerce na variedede-copa, passando pelo vigor, produção, qualidade dos frutos, nutrição e resistência a pragas e doenças. Para que essas características sejam atingidas, outras espécies e variedades têm sido experimentadas e recomendadas, numa tentativa de diversificação racional, visando a maior segurança para a citricultura, conforme salienta Pompeu Júnior (1991).

As tangerineiras constituem um grupo de plantas de particular interesse como porta-enxertos, pois, além da tolerância natural ao vírus da tristeza e ao declínio dos citros, induzem a produção de frutos de boa qualidade. Ressalta-se que a tangerineira- 'Cleópatra' é considerada suscetível à anomalia "declínio" (Muller et al., 1997). Dois exemplares bem conhecidos no Brasil são as tangerineiras 'Cleópatra' e 'Sunki', que também podem ser agrupadas como "microtangerinas". O termo microtangerinas proposto refere-se ao pequeno tamanho das folhas, flores e especialmente frutos, constituindo-se num grupo aparentemente homogêneo.

Devido à permanente necessidade de diversificação de variedades porta-enxertos e ao crescente interesse pelas microtangerinas, realizou-se o presente trabalho objetivando a caracterização física e morfológica dos frutos, de forma a propiciar maior conhecimento desse grupo de plantas e oferecer subsídios para futuras pesquisas.

\section{O grupo das microtangerinas}

Segundo Hodgson (1967), as tangerineiras originaram-se, provavelmente, no Nordeste da Índia ou no Sudeste da China. Esse autor refere-se à existência de uma forma primitiva chamada $C$. indica Tanaka, nas florestas de Assã, além da ocorrência de numerosos híbridos e outras formas não localizadas em outras partes do mundo.

O termo microtangerinas aqui referido compreende as espécies e variedades de Citrus spp., caracterizadas por apresentar folhas,

\footnotetext{
1 (Trabalho 055/2001). Recebido: 28/02/2001. Aceito para publicação: 06/09/2002.

Parte integrante da Tese de Doutorado do primeiro autor.

2 Pesquisador - Departamento de Fitotecnia e Fitossanidade/UEMA, Caixa Postal 6.006 - Cohab, 65.051-970, São Luís - MA. E-mail: gusmao@elo.com.br. 3 Pesquisador - Centro de Citricultura Sylvio Moreira/IAC, Caixa Postal 04, 13.490-970, Cordeiróplis - SP.
} 
flores e especialmente frutos pequenos, sendo os frutos normalmente ácidos. Essa denominação aproxima-se da expressão "small - fruited mandarins" usada por Hodgson (1967). De acordo com o citado autor, tendo por referência principalmente Tanaka (1954), "as tangerineiras de frutos pequenos" são compostas de 16 espécies, sendo que sete destas têm importância horticultural.

Donadio et al. (1998), em recente publicação Tangerinas ou Mandarinas, referem-se ao termo microtangerinas, as quais são representadas por várias espécies e usualmente utilizadas como porta-enxertos. Baseado na classificação hortícola das tangerineiras proposta por Hodgson (1967), Pio (1997) refere-se ao grupo das microtangerinas como "tangerineiras de pequenos frutos", citando entre elas as conhecidas 'Cleópatra' e 'Sunki'.

Os dois sistemas taxonômicos mais importantes e amplamente aceitos para citros, com relativas divergências, foram organizados por Swingle (1943) e Tanaka (1954). O primeiro reconheceu 16 espécies de Citrus, enquanto o segundo reconheceu 159 espécies válidas. Para as tangerineiras, Tanaka (1954) reconheceu um total de 36 espécies, divididas em 5 grupos taxonômicos. Destas 36 espécies, Swingle (1967) reconheceu somente três: $C$. tachibana (Japão), $C$. indica (Índia) e $C$. reticulata, na qual posicionou todas as outras.

Em estudos recentes desenvolvidos no Brasil a respeito da diversidade genética entre as tangerineiras, Coletta Filho et al. (1998), utilizando marcadores moleculares tipo RAPD, sustentam a classificação das tangerinas como espécie única (C. reticulata Blanco) proposta por Swingle (1943). Esses autores propõem, ainda, que as tangerineiras são compostas de diferentes grupos taxonômicos - formados de indivíduos geneticamente diferentes e um grande número de híbridos - pertencentes à referida espécie.

Entre as variedades do grupo das tangerineiras, as de importância como porta-enxerto são as microtangerinas 'Cleópatra' $(C$. reshni Hort. ex Tan) e 'Sunki' (C. sunki Hort. ex Tan). Além destas, outras cinco espécies têm importância horticultural: $C$. amblycarpa ('Nasnaran'), $C$. pectinifera (C. depressa), C. kinokuni, C. oleocarpa ('Timkat'), C. lycopersicaeformis ('Heennaran').

\section{MATERIAL E MÉTODOS}

O estudo foi conduzido no Departamento de Horticultura da Faculdade de Ciências Agronômicas (FCA/UNESP), Câmpus de Botucatu. As variedades de microtangerinas avaliadas foram provenientes do Banco Ativo de Germoplasma de Citros (BAG-Citros) da FCA/ UNESP, Botucatu e do BAG-Citros do CCSM/IAC, Cordeirópilis-SP. Incluiu-se também o limoeiro- 'Cravo' como testemunha.

A classificação taxonômica das espécies usadas está resumida na Tabela 1, de acordo com os dois principais sistemas propostos para as plantas cítricas: Swingle (1943) e Tanaka (1954).

As tangerineiras colhidas em Botucatu foram: 'Cleópatra' (clones CLE 1 e CLE 352); 'Sunki' (SUN 1, SUN 375); 'Suen Kat' (KAT 418); 'Pectinífera' (PEC); 'Nasnaran' (NAS 1 e NAS 2); 'Sun Chu Sha Kat' (KAT e KAT 1004); 'Crenatifolia' (CRE); e o limoeiro- 'Cravo' (clone Limeira). Do CCSM foram colhidas as tangerineiras 'Cleópatra' 352 (199), 'Sunki' 375 (200), 'Suen Kat' 418 (202), 'Nasnaran' (705), 'Sun Chu Sha Kat' 1004 (234), 'Tachibana' (708) (TAC), 'Pectinífera' 372, 'Keraji' 461 (KER), 'Heennaran' (HEN) e limoeiro- 'Cravo'-Limeira. Entre parênteses, indica-se o novo número do acesso no BAG-Citros do CCSM. O 'Nasnaran' do CCSM, proveniente da Califórnia, corresponde ao clone NAS 2 de Botucatu.

Para a avaliação dos atributos físicos e morfológicos, foram colhidos frutos maduros em junho do BAG-Citros de Botucatu, em 1995 e 1996, e frutos coletados em julho de 1996, junto ao BAG-Citros do CCSM-IAC, em Cordeirópolis-SP. Para comparação, incluíram-se frutos de tangerineira-'Poncã', colhidos em 1996, em pomar comercial de 12 anos de idade da Fazenda Morrinhos - Companhia Agrícola Botucatu.

Foram colhidos 100 frutos de duas plantas por variedade, nos diferentes quadrantes da copa, que foram armazenados em sacos plásti- cos, em câmara fria, $\mathrm{a}+4{ }^{\circ} \mathrm{C}$. Os frutos foram então misturados em caixa de colheita e em seguida tomadas, ao acaso, quatro subamostras de 10 frutos cada, num total de 40 frutos por variedade.

Foram avaliados os seguintes atributos: peso médio, altura, largura e índice de conformação; forma do fruto, da base e do ápice; coloração e superfície da casca (epicarpo), conforme recomendação de IBPGR (1988), Cereda et al. (1984) e Pio et al. (1991). Após estas mensurações, os frutos foram seccionados transversalmente e determinaram-se a espessura da casca, número de gomos ou segmentos, número de sementes normais e abortadas, diâmetro do eixo ou columela, aderência da casca e cor da polpa. As medidas foram tomadas com auxílio de paquímetro.

TABELA 1 - Classificação das espécies de microtangerinas e outros citros utilizados no trabalho, de acordo com os sistemas de Swingle (1943) e Tanaka (1954).

\begin{tabular}{|c|c|c|c|c|c|}
\hline \multirow{2}{*}{$\begin{array}{l}\text { Variedade/ } \\
\text { Nome Comum }\end{array}$} & \multicolumn{2}{|c|}{ Sistema } & \multirow{2}{*}{$\begin{array}{c}\text { Grupo } \\
\text { Taxonônico }{ }^{1}\end{array}$} & \multirow{2}{*}{$\begin{array}{l}\text { Origem } \\
\text { Provável }\end{array}$} & \multirow[b]{2}{*}{ Convenção } \\
\hline & Swingle (1943) & Tanaka (1954) & & & \\
\hline A & & & & & \\
\hline 'Cleópatra' & C. reticulata Blanco & C. reshni Hort. ex Tan. & IV & Índia & CLE \\
\hline 'Sunki' & C. reticulata Blanco & C. sunki Hort. ex Tan. & IV & China & SUN \\
\hline 'Suen kat' & C. reticulata Blanco & C. sunki Hott ex Tan. & IV & China & KAT \\
\hline 'Crenatifolia' & C. reticulata Blanco & C. crenatifolia Lush. & III & $* *$ & CRE \\
\hline 'Pectiniffera' * & C. reticulata Blanco & C. pectinifera Tanaka & V & Taiwan & PEC \\
\hline 'Nasnaran' & C. reticulata Blanco & C. amblycarpa Ochse & V & Egito & NAS \\
\hline 'Sun Chu Shu Kat' & C. reticulata Blanco & ** & * & China & KAT \\
\hline B & & & & & \\
\hline 'Heennaran' & C. reticulata Blanco & C. lycopersicaeformis Hott. ex Tan & V & Índia & HEN \\
\hline 'Keraji' & C. reticulata Blanco & C. keraji Hort. ex Tan & II & Japão & KER \\
\hline 'Tachibana' & C. tachibana (Mak.). Tan. & C. tachibana Tanaka & IV & Japão & $\mathrm{TAC}$ \\
\hline C & & & & & \\
\hline 'Poncä' & C. reticulata Blanco & C. reticulata Blanco & III & Índia & PON \\
\hline Limoeiro-'Cravo' & C. reticulata var, austera Swing. & C. limonia Osbeck & . & Índia & CRA \\
\hline
\end{tabular}

${ }^{1}$ Cinco grupos taxonômicos de Tanaka para as tangerinas, assim distribuídos segundo Hodgson (1967): I - Euacrumen (C. nobilis Loureiro); II - Anisodora; III - Megacarpa; IV - Angustifolia; e V - Latifolia; * Espécie também aceita como $C$. depressa Hayata (Tanaka, 1954) e vulgarmente conhecida como 'Shekwasha' (Swingle, 1967); ** Informação não disponível.

\section{RESULTADOS E DISCUSSÃO}

Os dados obtidos estão apresentados na Tabela 2. Os valores referentes a peso e dimensões do fruto, auxiliam na própria denominação do grupo de plantas - microtangerinas - citadas por Hodgson (1967) como "small fruited mandarins", especialmente aquelas incluídas nos grupos IV e V propostos por Tanaka (1954).

As tangerineiras 'Sunki' e 'Nasnaran' são representantes do grupo com frutos de menores peso e dimensão (Tabela 2). C. tachibana, pertencente ao BAG-citros de Cordeirópolis, também se constitui numa típica microtangerina. Apenas para efeito de comparação, a tangerina'Poncã' tem um peso médio aproximadamente 10 vezes superior a NAS 1 e 9,6 vezes superior a SUN 375, embora o peso médio de 'Poncã', no presente trabalho $(165 \mathrm{~g})$, seja pouco superior ao descrito por Figueiredo (1991), que é de 138g.

Em um nível intermediário de peso de fruto, situam-se os clones de 'Cleópatra', CRE, PEC e HEN. Os clones de 'Sun Chu Sha Kat' e $C$. keraji (KER) apresentam frutos de peso e dimensão ligeiramente maiores. Com relação ao clone KAT 418, que originalmente é tido como um “tipo" de C. sunki e denominado 'Suen Kat' (Tabela 1) conforme Tanaka (1954), o mesmo apresentou peso, dimensão e número de sementes bem 
superiores aos outros clones de 'Sunki' (SUN 1 e SUN 375). Dessa forma, pelas características descritas, 'Suen Kat' apresenta-se mais relacionado botanicamente com as tangerineiras 'Cleopatra' e 'Sun Chu Sha Kat'. Hodgson (1967) adota como sinonímias para a espécie $C$. sunki, os termos 'Suen Kat' e 'Sun kat' uma mandarina azeda do Sul da China, enquanto Swingle (1943) se refere a 'Sün Kat' como C. reticulata var. austera, e que os tipos chamados 'Kat' seriam formas dessa variedade. Conforme relatou Swingle (1943) acerca da possível origem híbrida de 'Suen Kat' (C. sunki x C. reticulata) e pelos resultados obtidos neste trabalho - maiores peso e dimensão do fruto, número de sementes, taxa de poliembrionia (dados não publicados) e cujas características foram repetidas nos BAG-Citros de Botucatu e Cordeirópolis - propõese que 'Suen Kat' seja incluída como uma variedade de Citrus sunki e não somente um clone respectivo.

O número de sementes do fruto é, a princípio, uma condição fundamental para uma variedade constituir-se um bom porta-enxerto. As variedades que produziram maior número de sementes normais ou viáveis foram 'Cleópatra', 'Sun Chu Shu Kat', KAT 418 e 'Heennaran'. Produziram poucas sementes os clones de 'Sunki', e as variedades KER e TAC. Note-se que o baixo número de sementes nomais de 'Sunki' parece estar relacionado com o alto número de sementes abortadas (Tabela 2), enquanto o uso de clones polinizadores mais eficientes e identificados por Medina Filho et al. (1992) poderia atenuar essa deficiência. Para 'Nasnaran', o número de sementes por fruto, variando de 9 a 10, confirma os resultados obtidos por Gravina (1989).

Com relação à variação entre os dois locais de amostragem de frutos, chama atenção o clone KAT 1004, que apresentou maior peso de frutos e menor número de semente no BAG-Citros de Cordeirópolis, em relação a Botucatu (Tabela 2). Essa variação pode estar relacionada à alternância de produção que ocorre normalmente nas variedades de tangerineiras (Figueiredo, 1991), em anos consecutivos.

Os detalhes da forma do fruto, base e ápice e coloração da casca constam na Tabela 3. Verifica-se ser predominante a forma oblata, característica típica das tangerineiras (Pio, 1997). As tangerinas 'Sunki' e 'Nasnaran' apresentaram uma variação no formato (oblata-esférica), conforme está indicado pelos índices de conformação mais elevados. A base variou de truncada a côncava e o ápice de deprimido a truncado. A cor da casca predominante foi a laranja e suas variações, sendo amarela em 'Nasnaran' e 'Tachibana'.

TABELA 2 - Resultados médios da caracterização física de frutos de microtangerinas do BAG-Citros da FCA/UNESP - Botucatu e do CCSM/IAC - Cordeirópolis-SP, obtidos durante os anos de 1995 e 1996.

\begin{tabular}{|c|c|c|c|c|c|c|c|c|c|c|c|c|c|c|c|c|}
\hline \multirow{3}{*}{$\begin{array}{l}\text { Espécies/ } \\
\text { variedades }\end{array}$} & \multicolumn{8}{|c|}{ BAG-Citros - Botucatu* } & \multicolumn{8}{|c|}{ BAG-Citros - Cordeirópolis (1996) } \\
\hline & \begin{tabular}{|l|} 
Peso \\
\end{tabular} & \begin{tabular}{|l|} 
Altura \\
\end{tabular} & Largura & \begin{tabular}{|l|l|} 
Espessurura| \\
\end{tabular} & Diâmetro & Núm. & \begin{tabular}{|l|} 
N. sem. \\
\end{tabular} & \begin{tabular}{|l|} 
N. sem. \\
\end{tabular} & Peso & \begin{tabular}{|l|l|} 
Altura \\
\end{tabular} & \begin{tabular}{|l|} 
Largura| \\
\end{tabular} & \begin{tabular}{|l|l|} 
Espessurura| \\
\end{tabular} & Diâmetro & \begin{tabular}{|l|} 
Núm. \\
\end{tabular} & \begin{tabular}{|l|} 
N. sem. \\
\end{tabular} & $\begin{array}{l}\text { N. sem. } \\
\end{array}$ \\
\hline & (g) & $(\mathrm{cm})$ & (cm) & $\operatorname{casca}(\mathrm{mm})$ & colum. (mm) & segm. & normais & abort. & (g) & (cm) & (cm) & $\operatorname{casca}(\mathrm{mm})$ & colum. (mm) & \begin{tabular}{|l|} 
segm. \\
\end{tabular} & normais & abort. \\
\hline CLE 352 & 34,5 & 3,4 & 4,6 & 3,1 & 7,4 & 11,0 & 18,9 & 1,8 & 33,8 & 3,5 & 4,6 & 3,4 & 10,7 & 10,6 & 16,4 & 1,1 \\
\hline SUN 375 & 17,2 & 2,9 & 3,3 & 2,5 & 7,2 & 8,8 & 3,4 & 7,9 & 22,4 & 3,2 & 4,3 & 2,3 & 9,6 & 8,9 & 2,1 & 7,7 \\
\hline KAT 418 & 41,3 & 3,4 & 4,7 & 3,5 & 9,3 & 11,2 & 18,1 & 1,9 & 36,7 & 3,6 & 4,8 & 3,4 & 11,2 & 10,9 & 19,6 & 0,4 \\
\hline KAT 1004 & 44,8 & 3,6 & 5,0 & 3,5 & 13,7 & 10,5 & 14,4 & 1,1 & 62,2 & 3,4 & 4,5 & 3,1 & 13,4 & 10,3 & 7,7 & 0,3 \\
\hline NAS2 & 21,4 & 3,1 & 3,6 & 2,6 & 3,5 & 9,6 & 9,1 & 1,4 & 19,9 & 2,7 & 3,4 & 2,4 & *** & 9,5 & 11,7 & 0,9 \\
\hline PEC & 33,3 & 3,3 & 4,6 & 2,6 & 11,1 & 10,0 & 6,8 & 0,5 & 25,7 & 3,1 & 4,1 & 2,6 & 10,2 & 10,2 & 5,6 & 0,0 \\
\hline CLE 1 & 39,4 & 3,6 & 4,6 & 3,2 & 6,7 & 11,0 & 20,9 & 0,9 & $\cdot$ & . & . & . & - & . & . & . \\
\hline SUN1 & 19,2 & 2,7 & 3,4 & 2,1 & 7,7 & 8,9 & 3,7 & 6,9 & $\cdot$ & . & . & . & . & . & . & . \\
\hline KAT & 38,2 & 3,4 & 4,7 & 2,9 & 7,7 & 11,0 & 19,7 & 2,3 & $\cdot$ & . & . & . & - & . & . & . \\
\hline NAS1 & 16,9 & 2,9 & 3,4 & 2,6 & 3,1 & 9,7 & 10,6 & 1,5 & $\cdot$ & . & . & . & . & . & . & . \\
\hline $\mathrm{CRE}^{1}$ & 35,7 & 3,4 & 4,6 & 3,8 & 5,9 & 9,8 & 9,0 & 2,0 & $\cdot$ & . & . & . & . & . & . & . \\
\hline $\mathrm{TAC}$ & . & . & $\cdot$ & . & . & $\cdot$ & . & . & 22,6 & 3,1 & 4,1 & 2,2 & 10,3 & 8,7 & 4,2 & 1,7 \\
\hline KER & . & . & . & . & . & $\cdot$ & . & . & 70,5 & 4,7 & 5,7 & 3,7 & 12,0 & 9,4 & 3,0 & 0,6 \\
\hline HEN & . & . & $\cdot$ & . & . & $\cdot$ & . & . & 38,5 & 3,8 & 5,1 & 3,9 & 13,8 & 10,6 & 18,4 & 1,1 \\
\hline CRA & 97,7 & 5,2 & 5,6 & 3,2 & 6,6 & 8,7 & 13,1 & 2,9 & 106,6 & 5,6 & 5,8 & 3,6 & 8,1 & 9,0 & 12,1 & 1,7 \\
\hline $\mathrm{PON}^{2}$ & 165,2 & 6,7 & 7,7 & 3,8 & 22,6 & 9,6 & . & - & - & . & . & . & . & . & . & . \\
\hline
\end{tabular}

* Médias dos dados de 1995-96; ${ }^{1}$ Dados somente de 1995; ${ }^{2}$ Dados somente de 1996; ** Columela fechada (sólida).
TABELA 3 - Características morfológias e sensoriais de frutos de microtangerinas, do BAG-Citros da FCA/UNESP Botucatu e do CCSM/IAC - Cordeirópolis-SP.

\begin{tabular}{|c|c|c|c|c|c|c|c|c|}
\hline \multirow[b]{2}{*}{ Variedade } & \multicolumn{4}{|c|}{ Forma } & \multicolumn{2}{|c|}{ Coloração } & \multicolumn{2}{|c|}{ Casca } \\
\hline & Fruto & $\mathrm{IC}^{*}$ & Base & Ápice & Casca & Polpa & Superfície & Aderência \\
\hline CLE 1 & $o b l$ & 0,77 & tru & dep & lar & lar & rug - lev & $\bmod$ \\
\hline CLE 352 & $o b l$ & 0,74 & tru & dep & lar & lar & rug - lev & $\bmod$ \\
\hline SUN 1 & $o b l$ & 0,77 & tru & dep & lar - for & lar & lis & lev \\
\hline SUN 375 & obl - esf & 0,86 & tru & dep & lar - for & lar & lis & lev \\
\hline KAT 418 & obl & 0,73 & tru & dep & lar - cla & lar & lis & lev \\
\hline KAT & obl & 0,72 & côn & dep & lar -for & lar & lis & lev \\
\hline KAT 1004 & obl & 0,72 & côn & dep & lar - for & lar & rug - lev & lev \\
\hline NAS 1 & obl - esf & 0,85 & tru & tru & aml & aml & rug & for \\
\hline NAS 2 & obl - esf & 0,86 & tru & tru & aml & $\mathrm{aml}$ & rug & for \\
\hline PEC & $o b l$ & 0,71 & tru & dep & lar - cla & aml & lis & lev \\
\hline TAC & $o b l$ & 0,76 & tru & dep & aml & lar - cla & lis & frx \\
\hline KER & $o b l$ & 0,82 & tru & dep & lar - cla & aml & rug - lev & frx \\
\hline HEN & $o b l$ & 0,75 & tru & dep & lar - for & lar - for & rug - ond & frx \\
\hline CRA & esf & 0,93 & $\operatorname{cox}$ & tru & lar & lar & lis & for \\
\hline PON & obl-esf & 0,87 & on-col-pes & dep & lar & lar & lis & frx \\
\hline
\end{tabular}

* Índice de conformação (A/L) obl = oblata; esf = esferóide; tru = truncado(a); dep = deprimido(a); côn = côncava; $\operatorname{cox}=$ convexa; col = colarinho; pes = pescoço; lar =laranja; $\mathrm{aml}=$ amarela; for $=$ forte $;$ cla $=$ claro $;$ lev $=$ leve $;$ rug $=$ rugosa; lis $=$ lisa mod $=$ moderada; frx $=$ frouxa .

\section{CONCLUSÕES}

1 - As microtangerinas estudadas apresentaram características semelhantes quanto à forma oblata dos frutos e coloração predominante laranja. Citrus amblycarpa, C. sunki e C. tachibana produziram os frutos de menor tamanho e espessura de casca. Houve diferenciação na dimensão dos frutos e número de sementes normais e abortadas.

2 - O maior número de sementes por fruto foi encontado nas tangerineiras 'Cleópatra', 'Sun Chu Sha Kat', 'Suen Kat' e 'Heennaran', enquanto nos clones de 'Sunki' predominou um elevado número de sementes abortadas em detrimento do baixo número de sementes normais.

3 - Propõe-se considerar 'Suen Kat' (Kat 418) como uma variedade de Citrus sunki, por apresentar peso e dimensões do fruto e número de sementes por fruto significativamente superiores aos clones respectivos de 'Sunki' descritos.

\section{AGRADECIMENTOS}

Aos Pesquisadores Dr. Joaquim Teófilo Sobrinho, Dr. Jorgino Pompeu Júnior e à Dra. Rose Mary Pio, do Centro de Citricultura Sylvio Moreira (CCSM/IAC), Cordeirópolis-SP, pela cessão dos frutos colhidos no BAG-Citros e pela valiosa colaboração neste trabalho.

\section{REFERÊNCIAS}

CEREDA, E., SALIBE,A.A., FERREIRA, V.L.P. Caracterização de cultivares de laranja-doce Citrus sinensis (L.) Osbeck de baixa acidez. III-Frutos. In: CONGRESSO BRASILEIRO DE FRUTICULTURA, 7., 1984, Florianópolis. Anais...Florianópolis: 1984, SBF, v.2, p.(irreg.).

COLETTA FILHO, H.D.; MACHADO. M.A., TARGON, M.L.P.N., MOREIRA, M.C.P.Q.D.G., POMPEU JÚNIOR, J. Analysis of the genetic diversity among mandarins (Citrus spp.) using RAPD. Euphytic $a$, Dordrechet, v.1, p.1-7, 1998.

DONADIO, L.C., STUCHI, E.S., CYRILLO, F.L.L. Tangerinas ou 
mandarinas. Jaboticabal: Funep, 1998. 40p. (Boletim Citrícola, 5).

FIGUEIREDO, J.O. Variedades-copa de valor comercial. In: RODRIGUEZ, O. et al. Citricultura brasileira. 2.ed. Campinas: Fundação Cargill, 1991. v.1, p.228-64.

GRAVINA, T.A. Caracterísiticas viveristas en nueve portainjertos de citricos. Chapingo, v.13/14, p.62-3, 1989.

HODGSON, R.W. Horticultural varieties of citrus. In: REUTHER, W. et al. The citrus industry. Berckley: University of California, 1967. v.1, p.431-591.

IBPGR - INTERNATIONAL BOARD FOR PLANT GENETIC RESOURCES. Descriptors for citrus. Rome: FAO, 1988. 27p.

MEDINAFILHO, H.P.; BORDIGNON, R.; BALLVÉ, R.M.L.; SIQUEIRA, W.J., CARVALHO, M.R.T. Porta-enxertos híbridos de citros produzidos no Instituto Agronômico de Campinas. Laranja, Corderopólis, v.13, p.777-792, 1992.

MOREIRA, C.S., MOREIRA, S. História da citricultura no Brasil. In: RODRIGUEZ, O. et al. Citricultura brasileira. 2.ed. Campinas: Fundação Cargill, 1991. v.1, p.1-18.

MÜLLER, G.W., LARANJEIRA, F.F., POMPEU JÚNIOR, J., CRISTOFANI, M., JORGE, M.F. Epidemias de declínio dos citros em combinações com tangerina-Cleópatra como porta-enxerto no Estado de São Paulo. Fitopatologia brasileira, 22 v. suplemento, p.345, 1997.

PIO, R.M. Estudo de variedades do grupo das tangerinas: caracterização e avaliação dos frutos. 1997. 89f. Tese (Doutorado em Fitotecnia), Escola Superior de Agricultura Luiz de Queiroz, Universidade de São Paulo, Piracicaba, 1997
PIO, R.M., POMPEU JÚNIOR, J., BOAVENTURA, Y.M.S. Caracterização das laranjas doces Seleta, Sanguínea, Tomango e Taroco. Revista Brasileira de Fruticultura, Cruz das Almas, v.13, p.201-09, 1991.

POMPEU JÚNIOR, J. Porta-enxertos para citros. In: RODRIGUEZ, O. et al. (Ed.) Citricultura brasileira. 2.ed. Campinas: Fundação Cargill, 1991. v.1, p.265-80.

SALIBE, A.A. Importância do porta-enxerto na citricultura. In: ENCONTRO NACIONAL DE FRUTICULTURA, 5., 1978, Rio de Janeiro. Anais ... Rio de Janeiro: PESAGRO-RIO/SBF, 1978. 14p.

SWINGLE, W.T. The botany of citrus and its wild relatives of the orange subfamily. In: WEBER, H.J., BATCHELLOR, L.D. (Ed.) The citrus industry. Berckley: University of California, 1943. v.1, p.129-474.

SWINGLE, W.T. The botany of citrus and its wild relatives. In: REUTHER, W., WEBBER, H.J., BATCHELOR, L.D. (Ed.) The Citrus industry. 2.ed. Berckley: University of California, 1967. v.1 (III), p.190-430.

TANAKA, T. Species problems in citrus (Revisio aurantiocearum, IX). Tokyo: Japanese Society for the promotion of Science, 1954. 152p.

WEBBER, H.J. Cultivated varieties of citrus. In: WEBBER, H.J., BATCHELOR, L.D. (Ed.) The citrus industry. Berckley: University of California, 1943. v.1, p.475-642.

WUTSCHER, H.K. Citrus rootstocks. Horticutural Reviews, v.1, p.23769, 1979. 